\title{
Numerical analysis on transitions and symmetry-breaking in the wake of a flapping foil
}

\author{
Guo-Yi He · Qi Wang · Xing Zhang · Shu-Guang Zhang
}

Received: 24 May 2012 / Revised: 19 October 2012 / Accepted: 29 Ocotber 2012

(C)The Chinese Society of Theoretical and Applied Mechanics and Springer-Verlag Berlin Heidelberg 2012

\begin{abstract}
Flying and marine animals often use flapping wings or tails to generate thrust. In this paper, we will use the simplest flapping model with a sinusoidal pitching motion over a range of frequency and amplitude to investigate the mechanism of thrust generation. Previous work focuses on the Karman vortex street and the reversed Karman vortex street but the transition between two states remains unknown. The present numerical simulation provides a complete scenario of flow patterns from the Karman vortex street to reversed Karman vortex street via aligned vortices and the ultimate state is the deflected Karman vortex street, as the parameters of flapping motions change. The results are in agreement with the previous experiment. We make further discussion on the relationship of the observed states with drag and thrust coefficients and explore the mechanism of enhanced thrust generation using flapping motions.
\end{abstract}

Keywords Flapping motion - Immersed boundary method Wake $\cdot$ Symmetry-breaking

The project was supported by the Natural Science Foundation of Jiangxi Province (2010GZC0162).

\section{G.-Y. He $\cdot$ Q. Wang}

The School of Aircraft Engineering,

Nanchang Hangkong University,

330063 Nanchang, China

X. Zhang (凶)

LNM, Institute of Mechanics,

Chinese Academy of Sciences,

100190 Beijing, China

e-mail: zhangx@lnm.imech.ac.cn

S.-G. Zhang

The School of Transportation Science and Engineering,

Beihang University, 100083 Beijing, China

\section{Introduction}

Flapping motion is a common mode for animal flying and swimming. Especially, vertical flapping including both pitching and plunging could generate a horizontal thrust by the interaction of moving foils with surrounding flows. Since the flapping motion is critically important to the design of micro-air-vehicles (MAVs), there exist various experimental studies and numerical simulations for exploring the mechanism of thrust generation [1-6]. A simple model to understand the mechanism of flapping-generated-thrust is the Karman vortex streets (KVS) and the reversed Karman vortex streets (RKVS). Both KVS and RKVS can be observed in the wake behind flapping foils, where the former is associated with drag and latter with thrust. In fact, as either amplitudes or frequencies increase in pitching motions, the transition from the KVS to the RKVS can be observed. The present paper is devoted to the study of transition of flow patterns and the parameters in flapping motions associated with thrust generation.

It was first observed by Knoller [7] and Betz [8] that a flapping wing could generate thrust. Karman and Burgers [9] offered the first theoretical explanation of drag or thrust production based on the wake vortices, where the wake of the flow past bluff bodies is modeled by an infinite row of alternating vortices, commonly known as KVS. Bohl and Koochesfahani [10] experimentally studied the RKVS with various reduced frequencies. Ellenrieder et al. [11] explained the Strouhal number dependency of the wake vortex structure behind a translating airfoil. Meanwhile, many researchers investigated the RKVS in different situations, such as the wakes of fishes [12,13] in both experiments [14-16] and numerical simulations [16-21]. However, a clearly understanding of the transition mechanism between KVS and RKVS in the wake behind flapping foils was not available. Recently, Godoy-Diana et al. [22] experimentally investigated the transition mechanism from KVS to RKVS and dis- 
cussed the relation of the transition process with thrust generation. In this paper, we will numerically study the transition of flow patterns in the wake of flapping foils and investigate their relation with thrust generations.

We will use the immersed boundary method [23-27] to numerically simulate Godoy-Diana et al.'s experiment and investigate the transition process from the KVS to the RKVS and its relation with thrust generation. Moreover, the numerical simulation offers some details of flow patterns, which are not available in experiments, so that the complete scenarios on flow patterns can be observed. The rest of this paper is organized as follows: Section 2 describes the mathematical models for the flows around the flapping foils and the numerical method. Section 3 presents the numerical simulation results on thrust or drag under different Strouhal numbers and their relation to the flow patterns. Finally, we will summarize the results of the present study in Sect. 4 .

\section{Mathematical model and numerical method}

\subsection{A simple model for flapping foil}

The present numerical configuration is set up to simulate the experiment by Godoy-Diana et al. [22]. The geometry of flapping foil is shown in Fig. 1, the diameter of the semicircle $D$ is $5 \mathrm{~mm}$ and its chord length $c$ is $23 \mathrm{~mm}$. The foil oscillates in pitching with respective to the center of the semicircle. The control parameters are the oncoming uniform velocity $U$, the flapping frequency $f$ and the peak to peak amplitude $A$. The main non-dimensional parameters are the Reynolds number $R e$, the pitching amplitude $A_{D}$ and the Strouhal number $S t$, defined as $R e=U D / v, A_{D}=A / D$ and $S t=f D / U$. The pitching motion of foils is set as a sinusoidal function of time

$A_{\mathrm{f}}(t)=-A_{D} \sin (2 \pi f t)$,

where $A_{\mathrm{f}}(t)$ is the flapping amplitude varying with time.

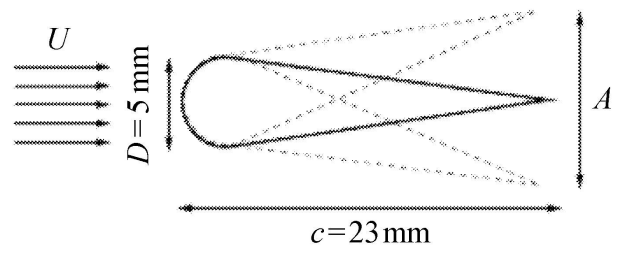

Fig. 1 Schematic views of the foil

\subsection{Governing equations}

The Navier-Stokes $(\mathrm{N}-\mathrm{S})$ equations for a 2-D incompressible flow are written as

$\frac{\partial \boldsymbol{u}}{\partial t}+\nabla(\boldsymbol{u u})=-\nabla p+\frac{1}{R e} \nabla^{2} \boldsymbol{u}+\boldsymbol{f}$, $\nabla \cdot \boldsymbol{u}=0$

where $\boldsymbol{u}$ is the velocity, $p$ is the pressure, and $\boldsymbol{f}$ is the external force. The boundary conditions are given as follows: At the inlet boundary, a uniform flow with velocity $U=1$, and the normal gradient of the pressure is set to be zero; At the outlet, the velocities are extrapolated from the interior and the normal gradient of the pressure is also zero; At the lateral boundaries, the gradient of tangential velocity is zero, the normal velocity is zero and the normal gradient of the pressure is also zero. The non-slip boundary conditions are applied to the surfaces of the foil where the fluid velocity is the same as that of the foil.

The computational region ranges from $-3 D$ to $19 D$ in the horizontal (or streamwise) direction ( $x$ direction), and from $-8 D$ to $8 D$ in the vertical direction with the origin at the centre of the semicircle. The number of uniform grids is $240 \times 120$ with $\Delta x=\Delta y=0.025$ in the rectangular region of $-D<x<5 D$ and $-1.5 D<y<1.5 D$, the grids are then stretched towards the boundary with the size growth ratio of 1.04 (see Fig. 2). The time step is 0.002 .

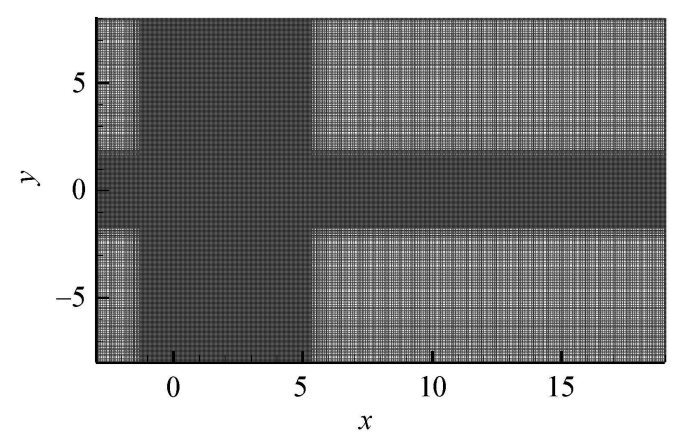

Fig. 2 Grid used for the present computation

\subsection{Numerical simulation}

We use the immersed boundary method to simulate the flow field around flapping foils, and force $\boldsymbol{f}$ in Eq. (2) will be calculated using the volume-of-fluid (VOF) method [28], The VOF method is an Eulerian scheme and uses the volume fraction $\Phi$ : The coefficient $\Phi$ is unity in the regions occupied entirely by solid phase and zero in regions occupied by fluid phase, and $0<\Phi<1$ in the regions partially occupied by either fluid and solid. Utilizing the VOF method, the solution procedure in the immersed boundary method can be summarized as follows:

(1) Solve the $\mathrm{N}-\mathrm{S}$ equations to obtain the intermediate velocity component $\tilde{\boldsymbol{u}}$

$$
\begin{aligned}
\frac{\tilde{\boldsymbol{u}}-\boldsymbol{u}^{n}}{\Delta t}= & -\frac{3}{2} \nabla_{h}(\boldsymbol{u} \boldsymbol{u})^{n}+\frac{1}{2} \nabla_{h}(\boldsymbol{u})^{n-1} \\
& -\nabla_{h} p^{n}+\frac{1}{2 \operatorname{Re}} \nabla_{h}^{2}\left(\boldsymbol{u}^{n}+\tilde{\boldsymbol{u}}\right) .
\end{aligned}
$$


(2) Calculate the volume forces by VOF

$\boldsymbol{f}=\Phi\left[\boldsymbol{u}_{\mathrm{rig}}^{n+1}-\tilde{\boldsymbol{u}}\right] / \Delta t$,

where $\boldsymbol{u}_{\text {rig }}$ is the velocity of the foil.

(3) Correct the intermediate velocity using the volume forces

$\frac{\boldsymbol{u}^{*}-\tilde{\boldsymbol{u}}}{\Delta t}=\boldsymbol{f}$.

(4) Calculate the intermediate velocity $\boldsymbol{u}^{* *}$, compute the pressure and update the fluid velocity $\boldsymbol{u}^{n+1}$

$\frac{\boldsymbol{u}^{* *}-\boldsymbol{u}^{*}}{\Delta t}=\nabla_{h} p^{n}$

$\nabla_{h}^{2} p^{n+1}=\frac{\nabla_{h} \cdot \boldsymbol{u}^{* *}}{\Delta t}$,

$\frac{\boldsymbol{u}^{n+1}-\boldsymbol{u}^{* *}}{\Delta t}=-\nabla_{h} p^{n+1}$.

Here, the projection method is used to decouple the solution of velocity and that of pressure. In time advancing, the Adams-Bashford and the Crank-Nicholson schemes are used for the convective and the diffusive term, respectively. The spatial discretization is based on the finite volume approach.

The immersed boundary code has been developed by our research group. To validate the code, one testing case is implemented. In this numerical example, we investigate the laminar flow induced by the harmonic in-line oscillation of a circular cylinder. According to Ref. [28], the key parameters are $R e=U_{\max } D / v=100, K C=U_{\max } /(f D)$, where $U_{\max }$ is the maximum velocity of the cylinder motion; $D$ denotes the diameter of the cylinder and $f$ is the frequency of the oscillation. The translational motion is given by the harmonic oscillation of $x(t)=-A_{\mathrm{c}} \sin (2 \pi f t)$, where $A_{\mathrm{c}}$ denotes the amplitude of the cylinder motion. Figure 3 shows the time history of the in-line force. From this figure, it is seen that the present numerical result is in agreement with that of Ref. [29].

For studying the propulsive performance of flapping

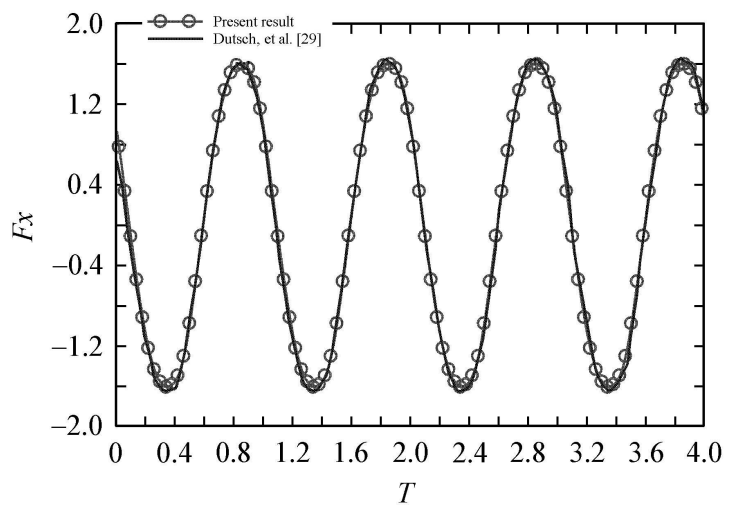

Fig. 3 Time history of the in-line force airfoils, we will calculate the drag coefficient defined as

$C_{\mathrm{d}}=\frac{2}{\rho U^{2} L} \int F_{1} \mathrm{~d} s$,

where $F_{1}$ is the force acting on the airfoil surface in the streamwise direction.

We performed simulations of the case with $R e=255$, $S t=0.22$ and $A_{D}=1.07$, using different time steps and grid resolutions. For the time step of 0.002 and 0.001 , the drag coefficients equal to 0.552 and 0.563 , respectively. For the grid width of 0.025 and 0.01 , the drag coefficients equal to 0.552 and 0.549 , respectively. The differences in the results obtained by using different time steps and mesh resolutions are sufficiently small.

\section{Numerical results}

The parameters in the present simulation are taken from the experiment by Godoy-Diana et al. [22]: the Reynolds number $R e=255$, the uniform oncoming flow $U=1$ and fluid density $\rho=1$. We will take different Strouhal numbers $S t$ and flapping amplitudes $A_{D}$ to study how the flow patterns are dependent on these parameters and compare the results obtained with the previous experiments of Godoy-Diana et al. [22].

Figure 4 is plotted for a fixed $S t=0.22$ and different values of $A_{D}$, equal to $0.36,0.71,1.07,1.77$ and 2.80 , respectively. The left column is for vorticity contours, the middle one for time averaged streamwise velocity contours and the right one for the time averaged streamwise velocity component in the near wake. The first row at $A_{D}=0.36$ is the typical case of low-amplitude pitching which produces a KVS, and the mean flow is a typical wake profile which characterizes drag. By increasing the amplitude $A_{D}$ to 0.71 , the vortices of alternating signs are aligned with the symmetry line of the wake. When the amplitude increases to $A_{D}=1.07$, the rotation direction of vortices changes and the flows in wakes transit from KVS to RKVS, and the mean flow is a typical jet profile which characterizes thrust. By further increasing amplitude to 1.77 , the vortex streets start to become deflected and asymmetric to the center line. As the amplitude increases to 2.8 , a symmetry breaking is observed in the RKVS. These plots clearly demonstrate that as the flapping amplitude increases, the flow patterns transit from KVS to RKVS and finally become deflected RKVS with symmetrybreaking.

Similar scenarios can be found by fixing the amplitude to $A_{D}=1.07$ and taking different Strouhal numbers, $S t=0.10,0.22,0.30,0.40$ (see Fig. 5). It is also observed that the transition from RKVS to the deflected RKVS occurs when the Strouhal number St or flapping frequency $f$ is higher than a critical value.

The bifurcation diagram for parameters $S t$ and $A_{D}$ is plotted in Fig. 6, which indicates different regimes of flow patterns: The KVS is located in the lower area, while the RKVS is located in the upper-right area. The regime of 
aligned vortices is in between and the deflected RKVS is in the region of largest $S t$ and $A / D$. These parameter regimes are qualitatively in agreement with the experimental results.
The bifurcation diagram offers transition paths for different Strouhal numbers and flapping amplitudes.
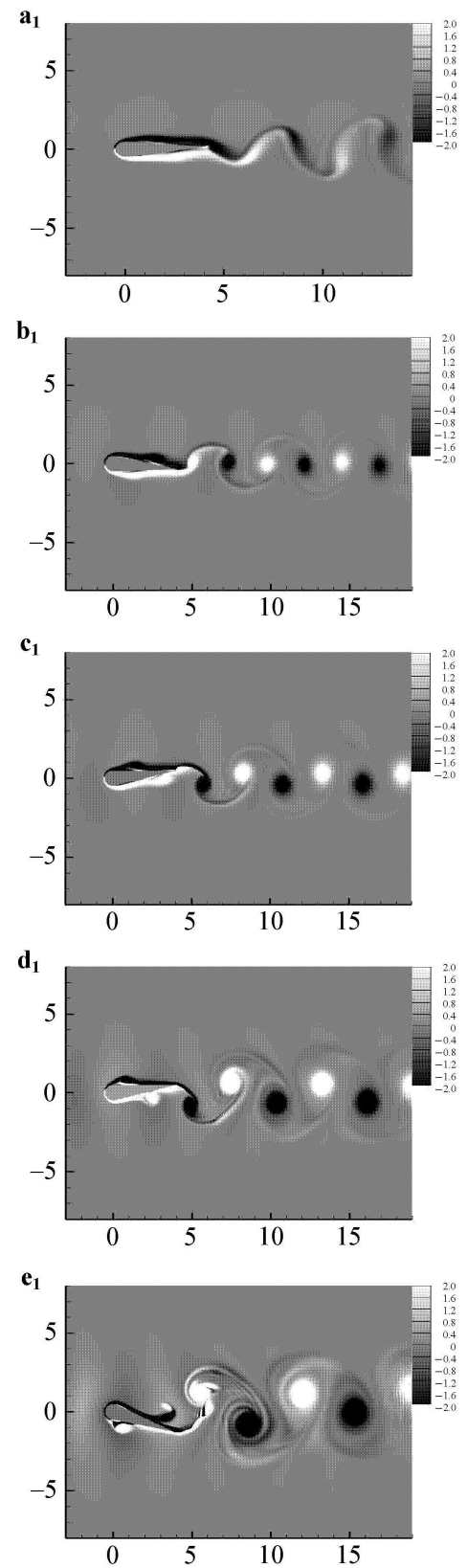

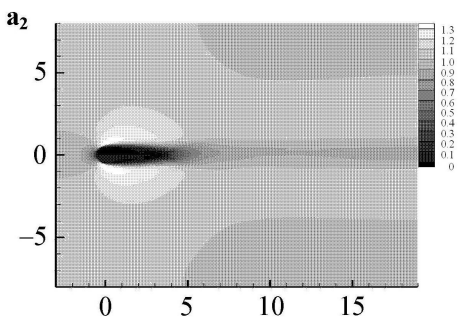

$\mathbf{b}_{2}$
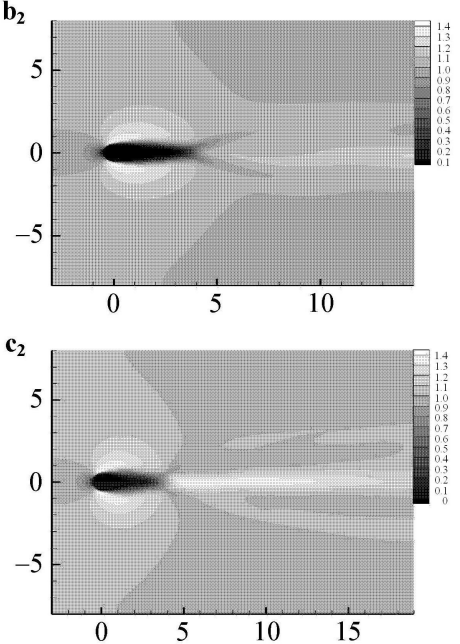

$\mathbf{d}_{2}$
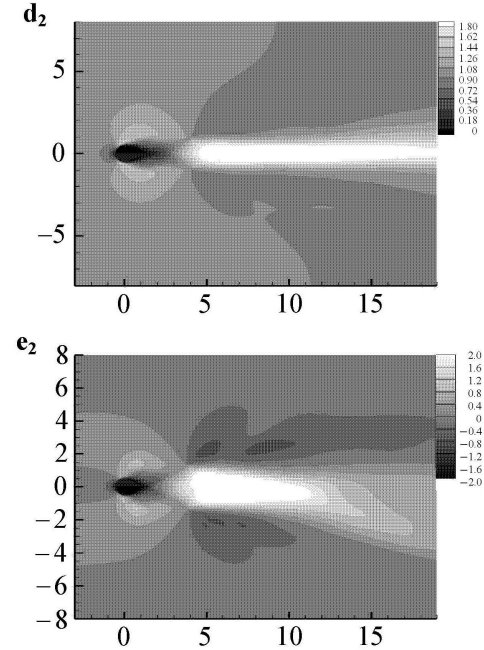

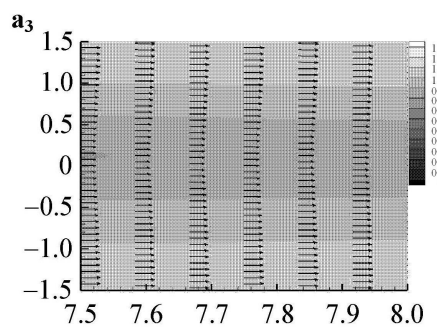

$\mathbf{b}_{3}$

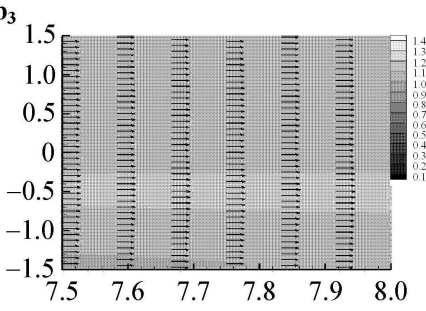

$\mathbf{c}_{3}$

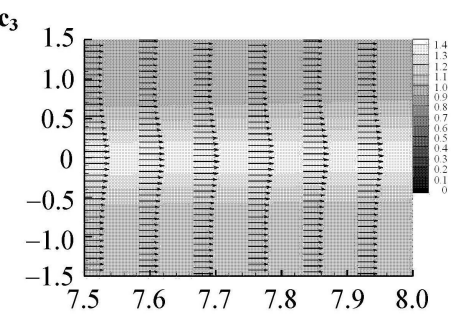

$\mathbf{d}_{3}$

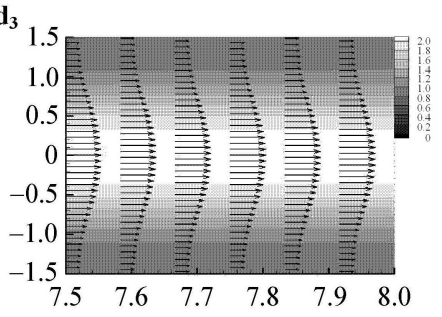

$\mathbf{e}_{3}$

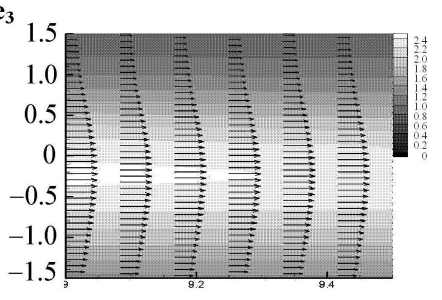

Fig. 4 Instantaneous vorticity contours (left column), time-averaged streamwise velocity contours (middle column) and time-averaged streamwise velocity component in the near wake (right column) for fixed $R e=255$ and $S t=0.22$, from top to bottom. a $A_{D}=0.36$; b $A_{D}=0.71 ; \mathbf{c} A_{D}=1.07 ; \mathbf{d} A_{D}=1.77$ and $\mathbf{e} A_{D}=2.8$

Figure 7 shows the dependency of the drag coefficient on the flapping amplitude $A_{D}$ for different Strouhal numbers. For the smallest $S t$, there is no thrust generation even for large $A_{D}$. As soon as $S t$ is larger than a threshold value, the flapping amplitude could affect thrust generation. For larger $S t$, the increase in flapping amplitude could significantly improve the thrust generation. 

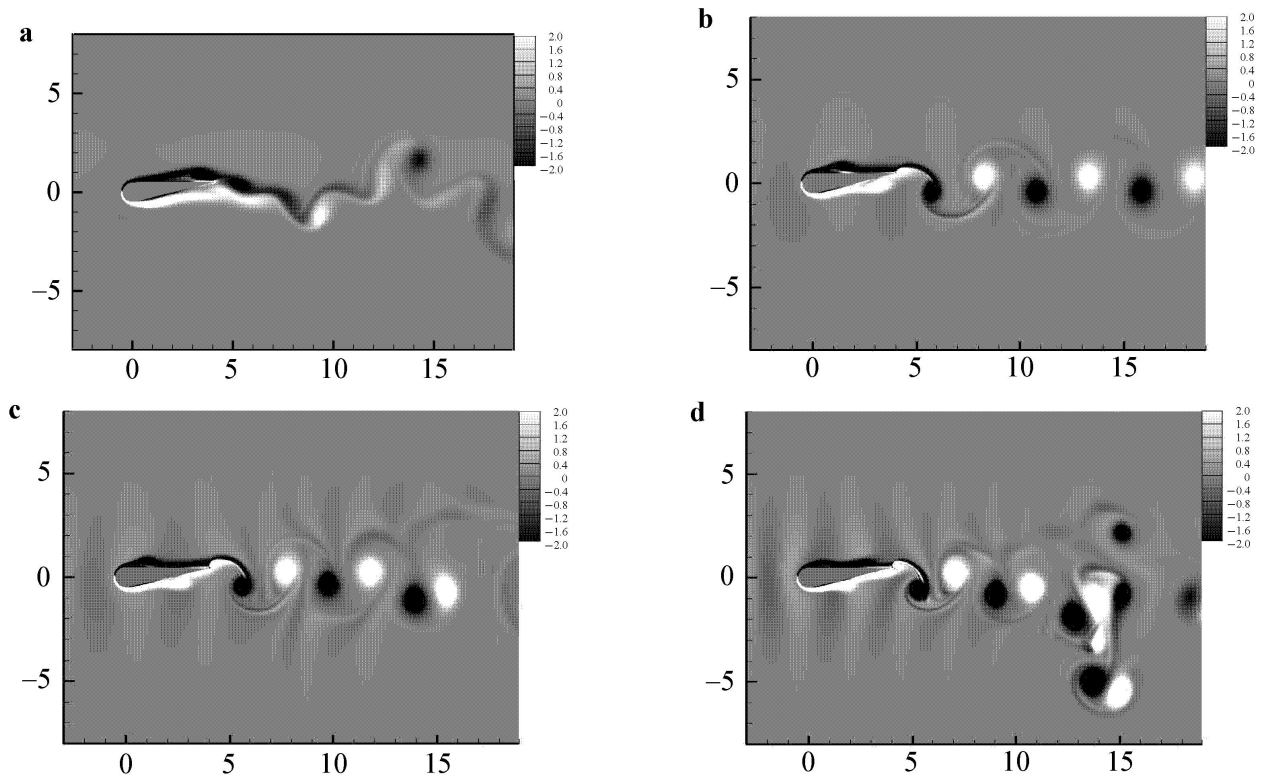

Fig. 5 Instantaneous vorticity contours for fixed $R e=255$ and $A_{D}=1.07$. a $S t=0.10 ; \mathbf{b} S t=0.22 ; \mathbf{c} S t=0.30$; $\mathbf{d} S t=0.40$

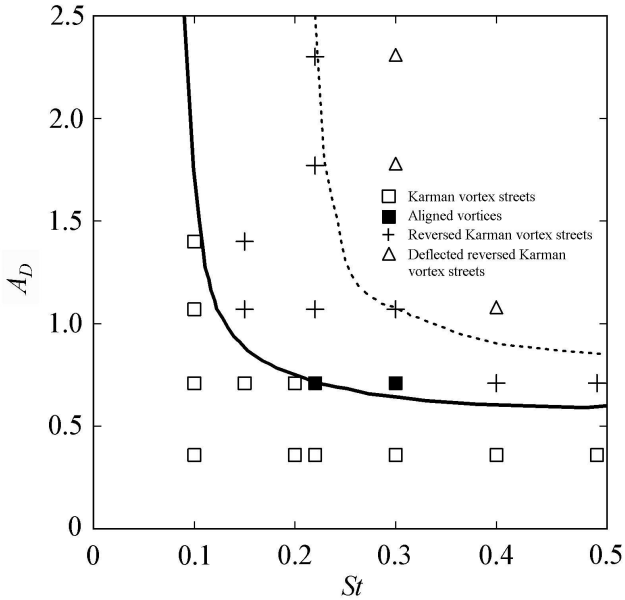

Fig. 6 The bifurcation diagram of $S t$ and $A_{D}$ for different flow patterns. Real line: transition between Karman vortex streets and reversed Karman vortex streets; Dotted line: transition between reversed Karman vortex streets and deflected reversed Karman vortex streets

\section{Conclusions}

In summary, the present numerical simulation shows complete transition scenarios of the wake behind a flapping foil: As either Strouhal number or flapping amplitude increases, the flow patterns can transit from KVS to RKVS via the aligned-vortices regime and finally become deflected RKVS when the Strouhal number and flapping amplitude are relatively large. The previous work has focused on KVS and RKVS. However, there is little work on the intermediate state (the aligned-vortices regime) and the ultimate state (de- flected RKVS). Godoy-Diana et al.'s experiment [22] first showed such scenarios and the present numerical simulations confirm the physical process with detailed observations. It is observed from the bifurcation diagram that there exist intermediate states and an ultimate state. The complete scenarios from KVS to RKVS offer an essential understanding of the thrust generation using flapping motions.

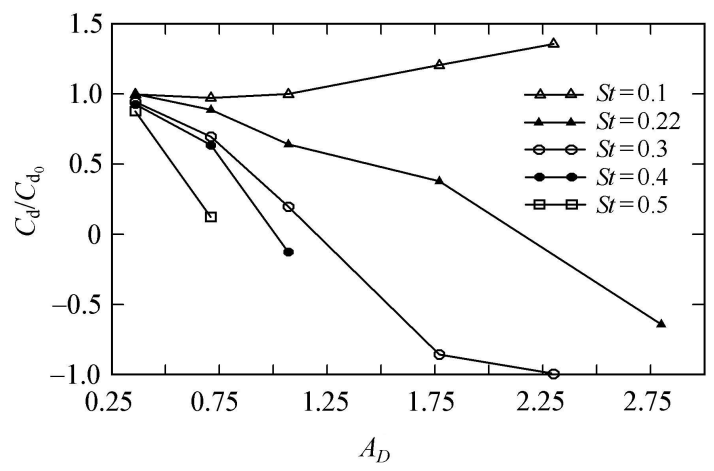

Fig. 7 The drag coefficients versus the flapping amplitudes $A_{D}$ for different St. $C_{\mathrm{d}_{0}}$ denotes the drag coefficient of non-flapping foil at zero angle of attack, $C_{\mathrm{d}_{0}}=0.861$

\section{References}

1 Anderson, J.M., Streitlien, K., Barrett, D.S., et al.: Oscillating foils of high propulsive efficiency. J. Fluid Mech. 360, 4-72 (1998)

2 Buchholz, J.H.J., Smits, A.J.: On the evolution of the wake structure produced by a low-aspect-ratio pitching panel. J. Fluid Mech. 546, 43-443 (2006) 
3 Buchholz, J.H.J., Smits, A.J.: The wake structure and thrust performance of a rigid low-aspect-ratio pitching panel. J. Fluid Mech. 603, 331-365 (2008)

4 Couder, Y., Basdevant, C.: Experimental and numerical study of vortex couples in two dimensional flows. J. Fluid Mech. 173, 225-251 (1986)

5 von Ellenrieder, K.D., Parker, K., Soria, J.: Flow structures behind a heaving and pitching finite-span wing. J. Fluid Mech. 490, 129-138 (2003)

6 von Ellenrieder, K.D., Pothos, S.: PIV measurements of the asymmetric wake of a two dimensional heaving hydrofoil. Exp. Fluids 44, 733-745 (2008)

7 Knoller, R.: Die Gesetze des Luftwiderstandes. Flug- und Motor-technik (Wien). 3, 1-7 (1909)

8 Betz, A.: Ein Beitrag zur Erkl"arung des Segelfluges. Zeitschrift fur Flugtechnik und Motorluftschiffahrt 3, 269-272 (1912)

9 von Karman, T., Burgers, J.M.: General Aerodynamic Theory Perfect Fluids, Aerodynamic Theory. Durand, W. F. edn. Division E 2, Julius-Springer, Berlin (1943)

10 Bohl, D.G., Koochesfahani, M.M.: MTV measurements of the vertical field in the wake of an airfoil oscillating at high reduced frequency. J. Fluid Mech. 620, 63-88 (2009)

11 von Ellenrieder, K.D., Parker, K., Soria, J.: Fluid mechanics of flapping wings. Experimental Thermal and Fluid Science 32, 1578-1589 (2008)

12 Wolfgang, M.J., Anderson, J.M., Grosenbaugh, M.A., et al.: Near-body flow dynamics in swimming fish. J. Exp. Biol. J. Exp. Biol. 202, 2303-2307 (1999)

13 Drucker, E.G., Lauder, G.V.: Locomotor function of the dorsal fin in teleost fishes: Experimental analysis of wake forces in sunfish. J. Exp. Biol. 204, 2943-2958 (2001)

14 Koochesfahani, M.M.: Vortical patterns in the wake of an oscillating airfoil. AIAA J. 27, 1200-1205 (1989)

15 Gopalkrishnan, R., Triantafyllou, M.S., Triantafyllou, G.S., et al.: Active vorticity control in a shear-flow using a flapping foil. J. Fluid Mech. 274, 1-21 (1994)

16 Jones, K.D., Dohring, C.M., Platzer, M.F.: An experimental and computational investigation of the Knoller-Betz effect.
AIAA J. 36, 1240-1246 (1998)

17 Molina, J., Zhang, X., Angland, D.: On the unsteady motion and stability of a heaving airfoil in ground effect. Acta Mech. Sin. 27,164-178 (2011)

18 Shyy, W., Liang, Y., Tang, J., et al.: Computational aerodynamics of low Reynolds number plunging, pitching and flexible wings. Acta Mech. Sin. 24, 351-373 (2008)

19 Dong, G.J., Lu, X.Y.: Numerical analysis on the propulsive performance and vortex shedding of fish-like traveling wavy plate. Int. J. Numer. Meth. Fluids 49, 1351-1373 (2005)

20 Sun, M., Wang, J.K., Xiong, Y.: Dynamic flight stability of hovering insects. Acta Mech. Sin. 23, 231-246 (2007)

$21 \mathrm{Wu}$, J.H., Sun, M.: Unsteady aerodynamic forces of a flapping wing. J. Exp. Biol. 207, 1137-1150 (2004)

22 Godoy-Diana, R., Aider, J.L., Wesfreid, J.E.: Transitions in the wake of a flapping foil. Physical Review E 77 (2008)

23 Mittal, R., Iaccarino, G.: Immersed boundary methods. Annual Review of Fluid Mechanics 37, 239-261 (2005)

24 Kim, J., Kim, D., Choi, H.: An immersed-boundary finitevolume method for simulations of flow in complex geometries. J. Comp. Phys. 171, 132-150 (2001)

25 Su, S.W., Lai, M.C., Lin, C.A.: An immersed boundary technique for simulating complex flows with rigid boundary. Computers and Fluids 36, 313-324 (2007)

26 Yang, X.L., He, G.W., Zhang, X.: Large-eddy simulation of flows past a flapping airfoil using immersed boundary method. Science China Physics, Mechanics and Astronomy 53, 1101$1108(2010)$

27 Yang, X.L., Zhang, X., Li, Z.L., et al.: A smoothing technique for discrete delta functions with application to immersed boundary method in moving boundary simulations. Journal of Computational Physics 228, 7821-7836 (2009)

28 Ravoux, J.F., Nadim, A., Haj-Hariri H.: An embedding method for bluff body flows: Interactions of two side-by-side cylinder wakes. Theoret. Comput. Fluid Dynamics 16, 433-466 (2003)

29 Dutsch, H., Durst, F., Becker, S., et al.: Low-Reynolds-number flow around an oscillating circular cylinder at low KeuleganCarpenter numbers. J. Fluid Mech. 360, 249-271 (1998) 